\title{
Autonome Fahrzeuge und autonomes Fahren aus Sicht der Nachfragemodellierung
}

\author{
Rita Cyganski
}

\section{Inhaltsverzeichnis}

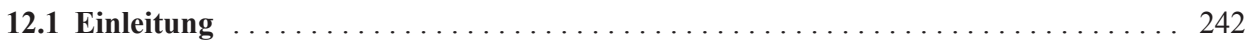

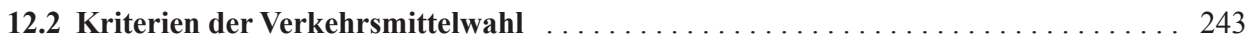

12.3 Die Verkehrsmittelwahl in angewandten Verkehrsmodellen . . . . . . . . . . . . 244

12.3.1 Eine kurze Einführung in die generelle Funktionsweise von Verkehrsnachfragemodellen . . . . . . . . . . . . . . . . . . . 244

12.3.2 Entscheidungskriterien in angewandten Modellen der Verkehrsmittelwahl . . . . 246

12.4 Welche Wirkung könnte die Einführung autonomer Fahrzeuge auf unser Verkehrsmittelwahlverhalten zeigen?

12.4.1 Der Autobahnpilot: ein Pkw mit dem besonderen Etwas

für Ausnahmesituationen? . . . . . . . . . . . . . . . . . . . . . . 248

12.4.2 Valet-Parken - nie mehr einen Parkplatz suchen? . . . . . . . . . . . . . . . . . . 248

12.4.3 Bequem und sicher ans Ziel mit einem vollautomatisierten Fahrzeug ... . . . 249

12.4.4 Das Vehicle-on-Demand - Zipcar on Steroids? . . . . . . . . . . . . . . . . 250

12.4.5 Das Auto der Zukunft: Konkurrenz für das Auto, das Taxi oder die Bahn? . . . . . 251

12.5 Welche Einsatzmöglichkeiten sehen Privatpersonen

für autonome Fahrzeuge? Erste Ergebnisse einer Befragung . . . . . . . . 251

12.5.1 Wer kann sich vorstellen, sein bisher bevorzugtes Verkehrsmittel zu ersetzen? . . 252

12.5.2 Worin sehen die Befragten den spezifischen Vorteil der autonomen Fahrzeuge? . 255

12.5.3 Was machen wir heute und zukünftig unterwegs? . . . . . . . . . . . 256

R. Cyganski $(\square)$

Deutsches Zentrum für Luft- und Raumfahrt e.V. (DLR), Institut für Verkehrsforschung, Deutschland Rita.Cyganski@dlr.de 


\subsection{Autonome Fahrzeuge in der Nachfragemodellierung: Möglichkeiten}

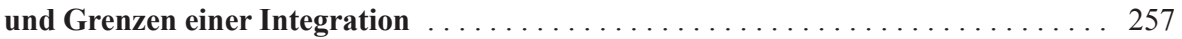

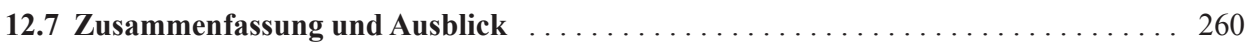

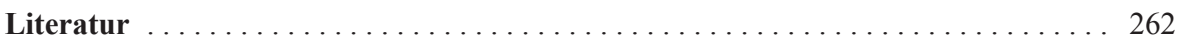

\subsection{Einleitung}

Luis G. Willumsen, einer der renommiertesten Wissenschaftler für die Verkehrsmodellierung, stellte 2013 auf einer Fachkonferenz fest: ,We can no longer ignore them [autonome Fahrzeuge], if planning horizon is 10+ years" [37]. Doch Arbeiten, die versuchen, die Auswirkungen der autonomen Fahrzeuge auf die Alltagsmobilität der potenziellen Nutzer und konkret deren Verkehrsmittelwahl zu antizipieren, finden sich erst selten [11], [19], [37]. Allerdings erlaubt ein Blick auf die individuellen Treiber unseres täglichen Mobilitätsverhaltens bereits jetzt vorsichtige Aussagen zu etwaigen Verhaltensänderungen durch die Einführung autonomer Fahrzeuge. Analogieschlüsse zur Nutzung bekannter Verkehrsmittel und ihre Übertragung in Verkehrsnachfragemodelle lassen erste quantitative Aussagen über Auswirkungen auf die Gesamtverkehrsnachfrage zu. Die Nachfragemodellierung ermöglicht es hierbei, zwischen unterschiedlichen räumlichen Kontexten und Nutzergruppen zu unterscheiden und verschiedene Szenarien zum Einsatz solcher Systeme zu evaluieren.

Ziel des Kapitels ist es, erste Ansätze zur Berücksichtigung autonomer Fahrzeuge bei der Verkehrsmittelwahl - auch „Modalwahl“ genannt - in Personenverkehrsnachfragemodellen zu skizzieren. Hierzu wird zunächst aufgezeigt, welchen Faktoren eine zentrale Rolle bei den individuellen Abwägungsprozessen zwischen unterschiedlichen Verkehrsmitteln zukommt. Der anschließende Abschnitt gibt eine kurze Einführung in die Funktionsweise von Verkehrsnachfragemodellen. Nachfolgend wird eruiert, welche Änderungen im Verkehrsmittelwahlverhalten durch die Einführung von autonomen Fahrzeugen möglich erscheinen. Differenziert nach unterschiedlichen Nutzungsszenarien wird erörtert, welche Eigenschaften der neuen Fahrzeuge, aber auch des räumlichen Kontextes und der etwaigen Nutzer selbst für die Wahrnehmung und Bewertung der Fahrzeuge von Bedeutung sein könnten und in welchem Konkurrenzverhältnis diese Fahrzeuge zu den bisherigen Verkehrsmitteln stehen. Zur Stützung der Ausführungen werden erste Ergebnisse einer Onlinebefragung zur Haltung gegenüber autonomen Fahrzeugen sowie ihres antizipierten Einsatzes vorgestellt. Abschließend wird aufgezeigt, welche Herausforderungen bei einer Integration autonomer Fahrzeuge in die modellgestützte Verkehrsnachfragebetrachtung bestehen und welche Erweiterungen der Modelle, aber auch der vorhandenen Datenbasis für eine adäquate Abbildung notwendig wären. 


\subsection{Kriterien der Verkehrsmittelwahl}

Die Frage, warum wir uns für bestimmte Verkehrsmittel entscheiden, ist Gegenstand einer Vielzahl an Publikationen aus unterschiedlichsten Disziplinen. Einigkeit herrscht darüber, dass dem menschlichen Verkehrsverhalten und der Verkehrsmittelwahl eine Vielzahl an komplexen interdependenten Faktoren zugrunde liegt. Bühler (2011) folgend, lassen sich diese in vier Gruppen unterteilen: (1) sozioökonomische und demografische Faktoren, (2) kultureller Rahmen und individuelle Einstellungen, (3) Strukturen der Raumentwicklung und (4) politische Regelungen [6]. Ortùzar und Willumsen (2005) nennen neben personenbezogenen Kriterien vor allem Eigenschaften der beabsichtigten Fahrt und nicht zuletzt des Verkehrsangebotes bzw. der zur Verfügung stehenden Verkehrsmittel als ausschlaggebend [24].

Von besonderer Bedeutung bei der Wahl des Verkehrsmittels ist die Frage nach dem Zugang zu den Verkehrsmittelalternativen. Führerscheinbesitz, die individuelle Einkommenssituation und damit eng verbunden der Pkw-Besitz gelten als wichtige Determinanten des Verhaltens. Substanzielle Investitionen wie die Anschaffung eines Pkw oder einer Zeitkarte für den öffentlichen Verkehr wirken langfristig auf die Verkehrsmittelwahl [31]. Deutliche Unterschiede im Modalwahlverhalten lassen sich nach Geschlecht, Erwerbsstatus, Haushaltsgröße und -struktur aufzeigen; einen besonderen Einfluss hat das Vorhandensein von Kindern im Haushalt [6], [24], [28]. Aber nicht nur objektiv messbare Kriterien spielen eine wichtige Rolle: Individuelle persönliche Lebensumstände, der eigene Lebensstil, Einstellungen zu den verschiedenen Verkehrsmitteln und zum Unterwegssein an sich, aber auch alltägliche Gewohnheiten und Routinen wirken auf die Entscheidung (vgl. [25], [27], [29], [33]). Die deutliche Wirkung der verkehrlichen Sozialisierung sowie sozialer und ökologischer Normen wird insbesondere bei lerntheoretischen, kognitiven und sozialpsychologischen Ansätzen betont (vgl. [2], [12], [27]). Sie zeigen deutlich, dass die individuellen Entscheidungen in der Praxis dem Idealbild eines unabhängig und rational entscheidenden Menschen oft nicht entsprechen.

Der Wohnstandort gilt als hauptsächlicher räumlicher Ankerpunkt der individuellen Verkehrsentscheidungen. Die dort bestehenden Raumstrukturen wirken direkt auf die Erreichbarkeit der angestrebten Ziele und die für Mobilität erbrachten Aufwände ([6], [9], [27], s. Kap. 11). So werden eine höhere Bevölkerungsdichte, eine starke Nutzungsmischung sowie die Nähe zum öffentlichen Verkehr mit geringerer Pkw-Orientierung und einer vermehrten Nutzung von Fuß und Rad in Verbindung gebracht; gleichzeitig treten Staus, Parkplatzknappheit sowie höhere Parkkosten verstärkt in dicht besiedelten Gebieten auf [6].

Die mit der Nutzung eines Verkehrsmittels einhergehenden finanziellen Kosten sind nicht zuletzt direkte oder indirekte Folgen politischer Regulierungen: Kraftstoff- und Haltungskosten, Maut- und Parkkosten beeinflussen die Bewertung des Pkw. Die zeitlichen Aufwendungen, insbesondere im Vergleich mit dem Zeitaufwand, der für die Alternativverkehrsmittel notwendig wäre, bilden ein weiteres wichtiges Wahlkriterium. Dabei gilt es, zwischen der Zeit, die im Vor- und Nachlauf einer Nutzung anfällt, also beispielsweise für 
den Weg zur Bushaltestelle und den Weg von der Bushaltestelle zum eigentlichen Ziel, und den eigentlichen Reisezeiten zu unterscheiden. Im Falle des öffentlichen Verkehrs lässt sich darüber hinaus zeigen, dass Warte- und Umsteigezeiten sowie die Anzahl der Umstiege maßgeblich die Bewertung beeinflussen [36]. Aber nicht nur instrumentelle Faktoren eines Verkehrsmittels, sondern auch die damit verbundenen symbolischen und affektiven Aspekte spielen eine große Rolle [33]. So wirken die vermeintliche oder reale Verlässlichkeit und Pünktlichkeit eines Verkehrsmittels ebenso wie die damit assoziierte Sicherheit, der empfundene Komfort, Fahrspaß oder auch die Flexibilität auf die Entscheidung für oder gegen ein spezifisches Verkehrsmittel [4], [24], [33].

Die Wahl eines Verkehrsmittels ist darüber hinaus auch abhängig vom Zweck des Weges. Dabei gilt es, nicht nur den einzelnen Weg, sondern den Kontext der gesamten Wegekette zu berücksichtigen, also alle Fahrten, die zwischen dem Verlassen der Wohnung und der Rückkehr vorgenommen werden [4]. Anzahl und Art der Begleitpersonen können dabei ebenso den Ausschlag für die Wahl eines Verkehrsmittels geben wie Transportnotwendigkeiten oder die zurückzulegende Distanz [16], [25], [33].

\subsection{Die Verkehrsmittelwahl in angewandten Verkehrsmodellen}

\subsubsection{Eine kurze Einführung in die generelle Funktionsweise von Verkehrsnachfragemodellen}

Verkehrsnachfragemodelle sind wichtige, etablierte Werkzeuge in verkehrsbezogenen Planungs- und Entscheidungsprozessen. Sie ermöglichen die Analyse der momentanen Verkehrssituation, die Prognose der zukünftigen Entwicklung der Verkehrsnachfrage oder die Untersuchung verschiedener potenzieller Entwicklungspfade anhand von Szenarien. Grundlage eines Verkehrsnachfragemodells stellt die vereinfachte, zweckspezifische Abbildung der Wirkungszusammenhänge zwischen dem Mobilitätsbedarf und seiner räumlichen Konkretisierung dar. Verkehrsmodelle sind mathematische Modelle, die hohe Anforderungen an Umfang und Detailtiefe der Eingangsdaten stellen und insbesondere in Bezug auf das menschliche Entscheidungsverhalten und das vorhandene Verkehrsangebot auf eine profunde empirische Datenbasis angewiesen sind.

Ziel der Personenverkehrsmodellierung ist die Abbildung all jener Entscheidungen von Individuen, die im Zuge einer geplanten Ortsveränderung getroffen werden. In der ersten Modellstufe, der Verkehrserzeugung, wird zunächst die Frage adressiert, wie viele Ortsveränderungen im Untersuchungsgebiet vorgenommen werden. Dazu wird für die Bevölkerung ermittelt, wie viele Wege oder Aktivitäten pro Person gemäß der Statistik an einem Tag zu erwarten sind. Die erzeugten Wege oder Wegeketten werden dabei nach Wegezweck unterschieden. Je nach Zweck des Weges erfolgt im nächsten Modellschritt, der Zielwahl oder Verkehrsverteilung, die Zuweisung eines Zielpunkts des Weges. Für die Kombination aus Ausgangs- und Zielort wird in der dritten Modellstufe, der Verkehrsmittelwahl oder Verkehrsaufteilung, zwischen den verschiedenen zur Verfügung stehenden Verkehrsmitteln 
abgewogen und eines ausgewählt. Im abschließenden Schritt, der Verkehrsumlegung oder Verkehrswegewahl, wird für die Fahrten gegebenenfalls der Startzeitpunkt bestimmt und festgelegt, welche Route genutzt wird, um vom Ausgangs- zum Zielort zu gelangen. Grundlage hierfür ist das sogenannte Angebotsmodell, in dem die zur Verfügung stehenden Verkehrssysteme detailliert abgebildet sind und so Attribute der verschiedenen Strecken -z. B. die Reisezeiten zwischen zwei Orten - ermittelt werden können. Als Ergebnis einer Verkehrsnachfragemodellierung liegen für den Untersuchungsraum die Ortsveränderungen der Bevölkerung sowie die resultierenden Verkehrsstärken für die einzelnen Verkehrsträger vor. Die einzelnen, hier vereinfacht sequenziell dargestellten Modellstufen finden in der Praxis oftmals simultan bzw. rekursiv Anwendung - insbesondere die Verkehrsmittel- und Zielwahl werden häufig kombiniert. Für eine vertiefende Darstellung der Verkehrsmodellierung sei auf [5] und [24] verwiesen.

Verkehrsnachfragemodelle werden in der Regel in mikroskopische und makroskopische Modellansätze unterschieden. Die Modellansätze unterscheiden sich sowohl hinsichtlich der notwendigen Informationen und Eigenschaften als auch der Modelllogik bei der Abbildung von Entscheidungszusammenhängen. In makroskopischen Nachfragemodellen, oftmals auch als Vier-Stufen-Modelle (Four Steps Models, FSM) bezeichnet, spiegeln sich die genannten vier Stufen der Verkehrsmodellierung direkt wider. Die Bevölkerung im Untersuchungsgebiet wird anhand ihrer soziodemografischen, verkehrlich relevanten Eigenschaften derart in Gruppen unterteilt, dass das Verhalten innerhalb einer Gruppe möglichst ähnlich ist, im Vergleich zu anderen Gruppen jedoch signifikant abweicht. Typische Einteilungen erfolgen hierbei anhand des Geschlechts, des Alters, des Erwerbsstatus sowie des Pkw-Besitzes. Eine Berücksichtigung des Haushaltskontextes findet in der Regel nicht statt (s. zu Details u. a. [18]). Im Verlauf der Modellierung werden alle Wege, die von Individuen dieser Gruppen generiert werden, gemeinsam und unabhängig von den sonstigen Ortsveränderungen des Tages modelliert. Für weiterführende Darstellungen der Funktionsweise makroskopischer Modelle sei auf [5] sowie [22] verwiesen.

Insbesondere in der regionalen Modellierung in den USA haben sogenannte aktivitätenbasierte oder mikroskopische Modellierungsansätze der Verkehrsnachfrage in den letzten Jahren an Bedeutung gewonnen. Im Vergleich zu makroskopischen Modellen legen diese einen stärkeren Fokus auf die individuellen Mobilitätsentscheidungen, die von Personen getroffen werden, und berücksichtigen in stärkerem Maße detaillierte Eigenschaften einer Person sowie des Haushaltskontextes. Die einzelnen Wege des Tages werden zu Touren zusammengefasst modelliert, die jeweils zu Hause starten und enden. Diese Form der Darstellung erlaubt die Berücksichtigung von Verhaltensabhängigkeiten zwischen einzelnen Aktivitäten und Entscheidungen. Im Zuge einer Mikrosimulation werden die Wegeketten für alle Personen im Untersuchungsgebiet einzeln berechnet und ergeben so das Gesamtbild der Verkehrsnachfrage. Vertiefte Informationen zur Funktionsweise aktivitätenbasierter Modellierungsansätze finden sich in [7], [10] und [21]. 


\subsubsection{Entscheidungskriterien in angewandten Modellen der Verkehrsmittelwahl}

Der Verkehrsmittelwahl kommt eine herausragende Bedeutung bei der Nachfragemodellierung zu, sind ihre Resultate doch in hohem Maße planungs- und politikrelevant [24]. Zum Einsatz kommen in der Regel diskrete Wahlmodelle oder eng verwandte Methoden, die auf der Annahme eines streng rationalen Entscheidungsverhaltens basieren. Dabei handelt es sich um statistische Kausalmodelle, mit deren Hilfe Aussagen zu den jeweiligen Einflussfaktoren und ihre Einflussstärke auf die Entscheidung hergeleitet werden können. Geht man davon aus, dass sich der Gesamtnutzen einer Wahlalternative additiv aus den einzelnen Komponenten herleiten lässt, kann so der Nutzen einer Alternative berechnet und mit demjenigen der anderen zur Verfügung stehenden Verkehrsmittel verglichen werden. Die Wahrscheinlichkeit der Wahl einer Variante steigt mit ihrem relativen Vorteil gegenüber den sonstigen Optionen [20], [24]. Die spezifizierten Modelle können von unterschiedlicher Komplexität sein (vgl. beispielsweise [34]). Verkehrsmittelwahlmodelle, die in Nachfragemodelle integriert werden, berücksichtigen in der Regel jedoch nur wenige Variablen, welche die Eigenschaften der Verkehrsmittel, der Personen, der Wege sowie der Raumstruktur umfassen.

Bei der Beschreibung der zur Verfügung stehenden Verkehrsmittel nehmen die finanziellen und zeitlichen Kosten, die mit der Wahl einhergehen, die herausragenden Stellungen ein. Im Falle des Pkw werden hier vorrangig Verbrauchskosten berücksichtigt, seltener Kosten der Anschaffung und Haltung. Bei den Zeitkosten handelt es sich vor allem um die durchschnittliche Fahrzeit, seltener und vor allem im öffentlichen Verkehr auch um die $\mathrm{Zu}$ - und Abgangszeiten. Bei letzterem werden oftmals zusätzlich die Anzahl der Umstiege, die Warte- und Umsteigezeiten oder auch Bedienungsintervalle berücksichtigt. Elemente wie die Zuverlässigkeit der Reisezeit finden sich bisher vorrangig in analytischen Modellen [4]. Mittels einer verkehrsmittelspezifischen Konstante können darüber hinaus nicht weiter spezifizierbare Nutzenkomponenten aggregiert Berücksichtigung finden. Alter, Geschlecht und Erwerbsstatus sowie Führerschein- und Pkw-Besitz zählen zu den üblichen soziodemografischen Attributen in den Modellen. Seltener finden sich Einkommensvariablen, das Bildungsniveau sowie die Haushaltsgröße oder die Anzahl der Kinder. Insbesondere bei den soziodemografischen Eigenschaften ist es üblich, diese bei der Ermittlung des Nutzens mit anderen Attributen zu interagieren und so personengruppenspezifische Stärken des Einflusses abzubilden. Die Beurteilung eines Verkehrsmittels kann auch von dem Zweck des Weges abhängen, und so sind ebenfalls wegezweckspezifische Interaktionsterme nicht unüblich. Eine besondere Bedeutung kommt hier der Unterscheidung der Arbeitswege zu. Aber auch die Transportnotwendigkeit oder die häufige Begleitung bei spezifischen Wegezwecken wie Einkauf oder Reisen können entsprechende Differenzierungen bedingen. Einen weiteren Bereich bilden die Raum- und Kontexteigenschaften. So können offizielle Raumtypisierungen oder auch die Bevölkerungsdichte am Wohnort eingesetzt werden, um eine Unterscheidung zwischen ländlicheren und dicht besiedelten Gebieten vorzunehmen. In manchen Modellen finden sich darüber hinaus Faktoren zur Beschreibung der Parksituation, der Parkkosten oder etwaigen Mautgebiete. 
Verkehrsnachfragemodelle sind stark datengetrieben und setzen die Möglichkeit voraus, quantitative Aussagen zu Wirkungszusammenhängen treffen zu können. Mangels Daten können zahlreiche Faktoren, die als relevant für die Verkehrsmittelwahl bekannt sind, oft nur vereinfacht in den Modellen Berücksichtigung finden. Betroffen sind vor allem sogenannte ,weiche Faktoren“ wie Routinen, die wahrgenommene Bequemlichkeit und Verlässlichkeit eines Verkehrsmittels, der assoziierte Fahrspaß, das damit verbundene soziale Ansehen oder auch das individuelle Sicherheits- und Rückzugsbedürfnis. Aber nicht nur Einstellungsfaktoren, auch das Wissen über die Alternativen, der eventuelle Planungsaufwand und das Abstimmungsverhalten im Haushalt sind Beispiele für Aspekte, die bei der Verkehrsnachfragemodellierung trotz ihrer bekannten Relevanz bisher kaum Berücksichtigung finden (vgl. u. a. [35]).

Eine spezielle Herausforderung besteht darüber hinaus in der Abbildung neuer Verkehrsmittel. Die Liberalisierung des Fernbusverkehrs in Deutschland, die Einführung stationsungebundener Carsharing-Angebote oder auch die Markteinführung elektrisch angetriebener Pkw sind Beispiele für Modifizierungen bestehender Verkehrsmittelangebote, bei denen sich eine Vorhersage der Nutzung schwierig gestaltet und belastbare Zahlen zu den Wirkungszusammenhängen fehlen. Doch auch eine Abgrenzung der verschiedenen bestehenden Verkehrsmittel anhand ,harter“ Faktoren ist nicht immer einfach. Verkehrsmodelle wurden historisch vor allem zur Berechnung der Fahrleistung von Pkw eingesetzt. Noch 2012 konstatiert Bates, klassischerweise hätten Vier-Stufen-Modelle kaum mehr als eine Differenzierung zwischen ,privaten Modi“, d.h. Pkw-Nutzung, und „öffentlichen“ Modi enthalten und wären erst in letzter Zeit um weitere Aspekte wie beispielsweise die Unterscheidung zwischen Fahrer und Mitfahrer erweitert worden [5]. Auch eine Differenzierung der Pkw, beispielsweise nach Größe oder Antriebsart, findet erst in neuerer Zeit Eingang in die Modelle. Insbesondere für die Integration autonomer Fahrzeuge in die Betrachtung offenbaren sich hier große Herausforderungen - denn schließlich gilt es, ,das Auto" näher zu betrachten und eine deutliche Unterscheidung zwischen Fahren und Gefahrenwerden, dem eigenen und einem geliehenen Pkw oder auch einem Taxi zu ermöglichen.

\subsection{Welche Wirkung könnte die Einführung autonomer Fahrzeuge auf unser Verkehrsmittelwahlverhalten zeigen?}

Bevor der Frage nachgegangen wird, wie autonome Fahrzeuge in der Verkehrsmodellierung Berücksichtigung finden können, muss geklärt werden, wie sich die Einführung dieser Systeme auf die individuelle tägliche Mobilität auswirken könnte. Die potenzielle Nutzung autonomer Fahrzeuge oder auch neuer Mobilitätsangebote ist i. A. stark abhängig von ihren konkreten Einsatzmöglichkeiten und den Vorteilen, die sich im Vergleich zu momentan bereits verfügbaren Verkehrsmitteln bieten würden - von Faktoren also, die für die Bewertung der autonomen Fahrzeuge im Vergleich mit anderen Modi bei der Verkehrsmittelwahl relevant sind und somit - sofern möglich - Eingang in die Modelle finden sollten. Für die in Kap. 2 vorrangig unter technischen oder rechtlichen Gesichtspunkten beschriebenen 
Anwendungsfälle werden daher in diesem Abschnitt zunächst mögliche Nutzungsvarianten erörtert. Im Vordergrund stehen dabei Fragen nach einer Typisierung der voraussichtlichen Nutzer, der Wege- und Einsatzwecke, für die sich entsprechende Systeme besonders eignen, der Raum- oder Kontexteigenschaften, die eine Nutzung fördern, den relevanten Verkehrsmitteleigenschaften sowie der Verkehrsmittelsubstitution, die sich als Resultat ergeben könnte. Der Fokus der Ausführungen wird auf diejenigen Eigenschaften gelegt, die sich für eine Abbildung in den Verkehrsmodellen eignen.

\subsubsection{Der Autobahnpilot: ein Pkw mit dem besonderen Etwas für Ausnahmesituationen?}

Der Autobahnpilot stellt möglicherweise ein Einstiegsszenario in die Automatisierung dar, da die Fahraufgabe außer in „Ausnahmesituationen“ bestehen bleibt. Die zwei Hauptaspekte des Autobahnpiloten aus der Nutzerperspektive stellen die Entlastung des Fahrers sowie die Möglichkeit, die Zeit während des Fahrens anders verbringen zu können, dar.

Der Einsatz des Autobahnpiloten wird nur auf spezifischen Strecken möglich sein und damit vorrangig auf Überlandfahrten. Aus diesem Grund kann angenommen werden, dass er sich vor allem bei Fahrten mit längerer Dauer positiv auf die Wahrnehmung und Bewertung des Pkws auswirken würde. Die Nutzerbefragung von Continental zeigt des Weiteren deutlich, dass die Abgabe der Fahraufgabe insbesondere bei stressbelasteten oder als lästig empfundenen Situationen wie Staus oder Baustellen positiv konnotiert ist [8]. Nicht selten wird damit aber auch eine Einschränkung des Fahrvergnügens verbunden [1].

Die Möglichkeit einer veränderten, zumeist mit erhöhter Sinnhaftigkeit oder Produktivität assoziierten Zeitnutzung ist nach der verbesserten Verkehrssicherheit der wohl meistgenannte Vorteil autonomer Fahrzeuge (s. beispielsweise [23], [26], [30], [32]). Insbesondere bei beruflich oder privat stark eingebundenen Personen, bei Fahrten mit Kindern oder in Begleitung könnte dies zu einer positiveren Bewertung der Reisezeit führen - einem der wichtigsten Faktoren bei der Verkehrsmittelwahl in Modellen. Letztendlich könnte dies dazu führen, dass längere Fahrten mit öffentlichen Verkehrsmitteln, insbesondere für Personen, die diese Zeit aktiv nutzen, relativ betrachtet unattraktiver werden und der Pkw als bequemer wahrgenommene Alternative an Zuspruch gewinnt. Die Bedeutung der alternativen Zeitnutzung ist jedoch schwer zu bemessen: In der Continental-Studie gab z. B. nur rund ein Drittel der Befragten die Möglichkeit einer alternativen Zeitnutzung als attraktiv an [8].

\subsubsection{Valet-Parken - nie mehr einen Parkplatz suchen?}

Beim Valet-Parken handelt es sich quasi um einen nur geringfügig modifizierten „normalen" Pkw, bei dem die Fahraufgabe unverändert bestehen bleibt. Nur das Abstellen und das Vorfahren bei Nutzungswunsch übernimmt das Fahrzeug selbstständig innerhalb eines 
definierten Radius. Gemäß einer Erhebung von AutoScout24 würden knapp zwei Drittel der Umfrageteilnehmer diese Funktion gern nutzen und nie wieder selbst einen Parkplatz suchen. Bei Bewohnern urbaner Gebiete ist der Anteil erwartungsgemäß höher [1] - ist die Funktion doch vor allem in Gebieten mit einer geringen Anzahl privater Stellplätze, mit Parkdruck und weiten $\mathrm{Zu}$ - und Abgangswegen vorteilhaft (s. auch Kap. 11). Sie hilft damit Zeit- und gegebenenfalls auch Parkkosten [19] zu sparen und wirkt komfortsteigernd, vor allem bei Transportaufgaben, dem Beisein von Kindern oder bei eingeschränkter Mobilität. Wenngleich eine höhere Carsharing-Nutzung vor allem bei der Einführung von Vehicleson-Demand diskutiert wird (s. z. B. [11]), könnte der sinkende Zugangsaufwand beim Valet-Parken eventuell das Carsharing erleichtern. Als sichere Konsequenzen können eine substanzielle Verringerung der Parksuchverkehre und damit einhergehend eine Senkung der Reisezeiten in bisher betroffenen Gebieten angesehen werden.

\subsubsection{Bequem und sicher ans Ziel mit einem vollautomatisierten Fahrzeug}

Das vollautomatisierte Fahren gilt als „Königsklasse der Fahrerassistenzsysteme“ [3]. Zwar wird - zumindest vorläufig - der Fahrzeuglenkende wohl prinzipiell in der Lage sein müssen, die Fahraufgabe zu übernehmen, notwendig soll dies aber nur auf Wunsch sein. Verbunden ist dieses Fahrkonzept bei hinreichenden Durchdringungsraten mit der Vision einer sichereren, zuverlässigeren Individualmobilität, einer Verbesserung des Verkehrsflusses und damit letztlich geringeren Stau- und Reisezeiten sowie einer erhöhten Planbarkeit des Reiseaufwandes [3], [26], [30]. Gleichzeitig kann davon ausgegangen werden, dass Besitz- und Nutzungshemmungen gegenüber dem Pkw sinken, insbesondere bei unerfahrenen, unsicheren oder auch älteren Fahrenden. Der Pkw wird so beispielsweise auch bei schlechteren Fahrkonditionen wie Dunkelheit, unbekannten oder langen Strecken sowie schlechten Wetterbedingungen eine attraktivere Option (s. auch [11]). Als Folge davon ist mit einem Rückgang der Bringwege, der Nutzung des Taxis und des öffentlichen Verkehrs einerseits sowie einem Anstieg des Pkw-Besitzes andererseits zu rechnen.

Der aus Nutzersicht meistgenannte Vorteil entsprechender Fahrzeuge ist jedoch - analog zum Autobahnpiloten - die damit verbundene Hoffnung, ,Zeit geschenkt [zu bekommen], die ich ganz für mich nutzen kann“ [26]. Es wundert daher wenig, dass zumeist Berufspendler als Zielgruppe dieser Technologie hervorgehoben werden (s. beispielsweise [17]) - bietet das vollautomatisierte Fahren doch auch und gerade in urbanen, dicht befahrenen Gebieten eine sinnvolle Nutzung der Onboard-Zeit. Auch hier gilt, dass dieser Vorzug vor allem dann zu einer positiveren Bewertung des Pkw führen dürfte, wenn die im Auto verbrachte Zeit vorher als nicht produktiv wahrgenommen wurde und der Fahrspaß nicht im Vordergrund der Nutzung steht. So lässt sich zeigen, dass der Nutzungswunsch besonders hoch bei Personen ausfällt, die sich nicht als passionierte Fahrer bezeichnen [17]. 


\subsubsection{Das Vehicle-on-Demand - Zipcar on Steroids?}

Ein Szenario, das von einer insbesondere in urbanen Räumen in weiten Teilen für jedermann verfüg- und nutzbaren Flotte an Fahrzeugen ausgeht, würde sicherlich die weitreichendsten Auswirkungen auf das tägliche Verkehrsverhalten implizieren. Als konsequente Weiterführung des vollautomatisierten Fahrzeuges, jedoch ohne Übernahme einer Fahrfunktion, ermöglicht es die individuelle, unabhängige Mobilität auch für Personen ohne Führerschein und eigenen Pkw: Kinder, Alte, Mobilitätseingeschränkte, sensorisch Beeinträchtigte etc. (vgl. u. a. [19]).

Generell ist davon auszugehen, dass das Individualfahrzeug durch die damit gleichzeitig einhergehende Senkung der Zu- und Abgangszeiten insbesondere in Gebieten mit Stellplatzproblematik an Attraktivität gewinnen wird. Neben einer denkbaren Senkung der Parkkosten durch die Möglichkeit, das Fahrzeug auch in weiterer Entfernung zum eigentlichen Zielort abzustellen, ist mit einer deutlichen Verringerung sogenannter Bring- und Holwege sowie der Nutzung von Fahrdiensten und Taxis zu rechnen - eventuell bei gleichzeitiger Zunahme der Leerfahrten.

Als Folge der Einführung von Vehicle-on-Demand-Flotten wird gleichzeitig unisono eine substanziell niedrige Autobesitzquote erwartet [19], [37], die mit einem Boom des Carsharing einhergehen könnte: Silberg et al. (2012) sprechen mit Verweis auf das aus den USA stammende Carsharing-Unternehmen gar von „Zipcar on Steroids“ [30]. Insbesondere bei Zweitwagen erscheint es plausibel, dass diese durch entsprechende Angebote abgelöst würden, die gleichzeitig eine Anpassung des gewählten Fahrzeuges an die situative Beförderungs- und Parksituation oder auch an Wetterbedingungen ermöglichen (vgl. [17], [37]).

Dass von einer substanziellen Wirkung auf den individuellen Pkw-Besitz ausgegangen werden kann, erwarten Fagnant und Kockelman (2013): Gemäß ihrer Simulationsrechnungen für die USA könnte ein einzelnes Vehicle-on-Demand bis zu 13 private Pkw substituieren [11]. Arbeiten zum stationsgebundenen Carsharing beziffern die Substitutionsraten auf bis zu acht durch ein einzelnes Carsharing-Auto ersetzte private Pkw in Deutschland [15]. Da ein Vehicle-on-Demand durch den Wegfall des Zugangsweges einen größeren Einzugsbereich abdecken könnte, erscheinen diese Abschätzungen durchaus nicht unrealistisch. Ein Blick auf die Nutzerstruktur bestehender Carsharing-Angebote gibt erste Hinweise auf die potenziellen Nutzer von Vehicle-on-Demand-Angeboten: Insbesondere jüngere Personen mit überdurchschnittlicher Bildung und Einkommenssituation sowie einem ausgeprägten Interesse an ökologischen Themen nutzen in Deutschland stationsgebundene Carsharing-Angebote; die noch recht neuen stationsungebundenen Angebote werden vor allem von urbanen, männlichen Nutzern als flexible Verkehrsmitteloption bei kürzeren Wegen eingesetzt (vgl. [13], [14]). Die Nutzung von Vehicle-on-Demand-Angeboten als Alternative zum eigenen Pkw könnten so direkt zur Förderung eines multimodalen Verkehrsmittelwahlverhaltens oder zu einem Anstieg von Fahrgemeinschaften beitragen (vgl. [26]). Insbesondere vor dem Hintergrund einer positiver beurteilten Zeit im Fahrzeug ist jedoch auch ein Anstieg der Pkw-Nutzung und -Fahrleistung im Zuge der omnipräsenten 
Verfügbarkeit ([11], [37], s. Kap. 11) denkbar. Das Thema „Neue Mobilitätskonzepte“wird in Kap. 9 umfassend betrachtet.

Doch nicht nur der individuelle Pkw-Besitz könnte in einem solchen Szenario weitreichenden Änderungen unterworfen sein. Fahrdienste, Taxen und der öffentliche Verkehr könnten sich in einer verschärften Wettbewerbssituation zum Individualtransportmittel wiederfinden. Insbesondere im ländlichen Raum könnten Vehicle-on-Demand-Flotten einerseits als flexible, individuelle Zubringerdienste zum öffentlichen Nahverkehr dienen oder die bequeme Überwindung der „letzten Meile“ im öffentlichen Fernverkehr gewährleisten. Kritische Betrachtungen erlauben aber auch die These, dass sie die Versorgung mit öffentlichen Massenverkehrsmitteln nicht ergänzen, sondern langfristig sogar ganz ersetzen könnten: ,... eventually, mobility on demand may prove a better investment than new mass transit systems. “[17]: eine Einschätzung, die auch von Willumsen (2013) geteilt wird [37]. In diesem Fall lassen sich Annahmen, dass die individuell zurückgelegten Personenkilometer deutlich ansteigen könnten, sicherlich nicht von der Hand weisen.

\subsubsection{Das Auto der Zukunft: Konkurrenz für das Auto, das Taxi oder die Bahn?}

Die Analyse der bisher zur Verfügung stehenden Literatur zeigt, dass die Einführung autonomer Fahrzeuge sehr unterschiedliche Auswirkungen auf unsere tägliche Mobilität und unsere gewählten Verkehrsmittel haben könnte. Das autonome Fahren stellt somit nicht immer nur eine leichte Modifikation unseres altbekannten Pkw dar, durch die sich der Verkehrsfluss und die Reisezeiten verbessern und wahlweise Unterstützungsoptionen genutzt werden können, wie eine Grafik in der KPMG-Studie [17] suggeriert. Vielmehr unterscheiden sich die potenziellen Auswirkungen auf die individuell oder auch öffentlich zur Verfügung stehenden Verkehrsmittel enorm. Die weitreichenden Implikationen, die sich aus den unterschiedlichen Szenarien für das Gesamtverkehrssystem und die Nutzung des öffentlichen Raumes generell ergeben könnten, werden in Kap. 11 vertieft aufgegriffen. Deutlich zeigt sich in der vorangegangenen Literaturanalyse zu möglichen Auswirkungen auf die Verkehrsmittelwahl, dass die wahrnehmungs- und bewertungsrelevanten Eigenschaften in den verschiedenen Nutzungsszenarien sehr unterschiedlich sein können und somit eine differenzierte Betrachtung bei der Analyse einer möglichen Abbildung autonomer Fahrzeuge in der Verkehrsnachfragemodellierung erfordern.

\subsection{Welche Einsatzmöglichkeiten sehen Privatpersonen für autonome Fahrzeuge? Erste Ergebnisse einer Befragung}

Seit 2012 wurden vereinzelt quantitative Studien zum Thema des autonomen Fahrens durchgeführt [1], [8], [17]. Der Fokus der Erhebungen liegt in der Regel auf Einstellungs- und Akzeptanzfragen oder gewünschten Unterstützungsfunktionen. Mit einigen Fragen zur an- 
tizipierten Nutzung und alternativen Zeitverwendung ist die Mobilitätsstudie 2013 von Continental [8] hervorzuheben. Allen Studien ist jedoch gemein, dass sie keine Differenzierung der möglichen Ausprägungen des autonomen Fahrens berücksichtigen und nicht auf die Art der möglichen Verhaltensänderungen oder der potenziellen Einsatzzwecke abzielen.

Aus diesem Grund wurde im Juni 2014 eine Ergebung durchgeführt, die empirische Aussagen sowohl zu Fragestellungen dieses Beitrags als auch zur Ausgestaltung der Mensch-Maschine-Schnittstelle (s. Kap. 6) sowie zur Akzeptanz autonomer Fahrzeuge (s. Kap. 29) ermöglicht. Dabei handelt es sich um eine quasi-repräsentative Onlineerhebung, die sich auf die Einstellungen der Befragten bezieht und auf ihre Einschätzung zur Nutzung eines autonomen Fahrzeugs entsprechend der verschiedenen Nutzungsszenarien des Projektes. Die nach Geschlecht, Alter, Einkommen und Bildung geschichtete Stichprobe umfasst jeweils 250 vollständige Fragebögen pro Nutzungsszenario, insgesamt also 1000 Befragte. Der Datensatz erlaubt es, mittels quantitativer Methoden eine erste Einschätzung zur Haltung verschiedener Nutzergruppen zu autonomen Fahrzeugen und Mobilitätsangeboten zu erlangen und so die Ausführungen des vorangegangenen Abschnittes zu ergänzen. Ziel der Befragung aus Sicht der Nachfragemodellierung ist es vor allem zu ergründen, welche Unterscheidungen bei den Nutzern, den Wege- und Einsatzzwecken, aber auch den Verkehrsmitteleigenschaften für eine Abbildung autonomer Fahrzeuge in Verkehrsmodellen wichtig sind. Auch sollen mithilfe der Datenanalysen erste Hinweise darauf erlangt werden, mit welchen Verkehrsmitteln die autonomen Fahrzeuge aus heutiger Sicht in Nutzungskonkurrenz stehen könnten und wie sich die Reisezeitverwendung und -bewertung ändern könnte.

Der Fragebogen enthält zunächst Fragen zur Soziodemografie der Probanden, zu ihrem Kenntnisstand und ihrem Interesse am autonomen Fahren sowie zur bisherigen Nutzung von Assistenzsystemen. Anschließend wird die momentane Nutzung von und Einstellung gegenüber den zur Verfügung stehenden Verkehrsmitteln und die übliche Zeitverwendung bei ihrer Nutzung erhoben. Die Probanden beantworten darüber hinaus für jeweils eines der im Projektkontext verwendeten Nutzungsszenarien Autobahnpilot, autonomes ValetParken, vollautomatisiertes Fahrzeug sowie Vehicle-on-Demand (s. Kap. 2) vertiefende Fragen zur antizipierten Nutzung, zum ersetzten Verkehrsmittel, zur Einstellung gegenüber dem beschriebenen Fahrzeug, zum Bedürfnis nach Interventionsmöglichkeiten, zu verschiedenen Erlebnisaspekten und zu konkreten Gestaltungswünschen. Die Beschreibungen der Szenarien sind dabei kurz gehalten, um den Befragungsteilnehmern Platz für eigene Interpretationen zu gewähren. Ein tabellarischer Überblick der soziodemografischen Eigenschaften der Befragten sowie der Stichprobenstruktur findet sich in Kap. 6.

\subsubsection{Wer kann sich vorstellen, sein bisher bevorzugtes Verkehrsmittel zu ersetzen?}

Um die Wirkung autonomer Fahrzeuge auf die Verkehrsmittelwahl abbilden zu können, ist es besonders wichtig zu wissen, welche Verkehrsmittel von diesen autonomen Fahr- 


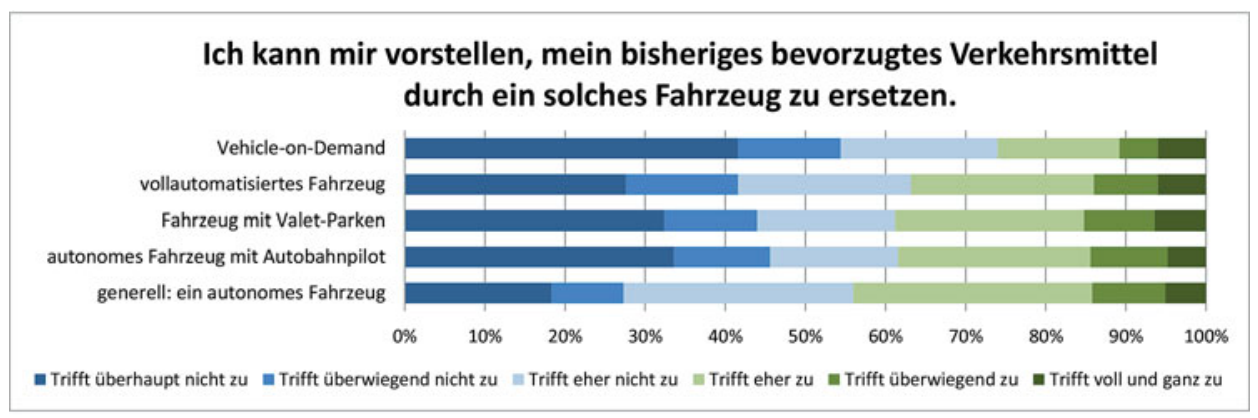

Abb. 12.1 Konstatierte Ersetzungsbereitschaft des bevorzugten Verkehrsmittels durch ein autonomes Fahrzeug generell sowie in den verschiedenen Nutzungsszenarien

zeugen substituiert werden könnten. Daher thematisiert die Erhebung in einer der ersten Fragen die grundsätzliche Bereitschaft, das bevorzugte Verkehrsmittel durch ein autonomes Fahrzeug zu ersetzen - zunächst ohne eine Differenzierung. Im weiteren Verlauf wird zusätzlich für die jeweiligen Nutzungsszenarien erhoben, ob die Befragten sich vorstellen könnten, ein entsprechendes Fahrzeug generell zu nutzen bzw. ihr bevorzugtes Verkehrsmittel durch ein autonomes Fahrzeug zu ersetzen. Die Ergebnisse wurden auch daraufhin untersucht, ob sich signifikante Unterschiede im Antwortverhalten der Befragten in Abhängigkeit von ihren soziodemografischen Eigenschaften und Einstellungen nachweisen lassen. ${ }^{1}$ Entsprechende statistische Kennwerte zeigen auf, dass bei einer Integration in die Modelle eine Unterscheidung des Verhaltens anhand dieser Kriterien vorgenommen werden sollte - die konkrete Stärke oder Richtung der Wirkung ist zunächst zweitrangig.

Die jeweiligen Antworten für den Ersetzungswunsch sind in Abb. 12.1 dargestellt und entsprechen in der Tendenz dem generellen Einsatzwillen (s. Kap. 31). Auffällig ist, dass die Mehrheit der Befragten sich eher nicht bis gar nicht vorstellen kann, einem autonomen Fahrzeug den Vorrang vor ihrem bisherigen Standardverkehrsmittel zu geben. Weniger als 15 Prozent der Befragten stimmen der Aussage zu, ein - wie auch immer geartetes - autonomes Fahrzeug überwiegend oder ganz als Ersatz nutzen zu wollen. Während bei der generellen Aussage der Anteil der unentschiedenen Befragungsteilnehmer sehr hoch ausfällt, sinkt der Zustimmungswert in den Szenarien deutlich und unterscheidet sich statistisch signifikant zwischen den einzelnen Szenarien. Mit nur rund einem Viertel positiver Antworten sticht das Vehicle-on-Demand dabei als besonders skeptisch betrachteter Anwendungsfall heraus. Dem Vollautomaten hingegen stehen die Befragten im Durchschnitt am positivsten gegenüber.

1 Als statistisch signifikant werden nachfolgend Unterschiede aufgeführt, die bei einem ChiQuadrat-Test nach Pearson Werte von 0,05 oder niedriger aufweisen. Bei einigen Variablen, insbesondere den Einstellungs- und Raumvariablen war ein Test aufgrund der Fallzahlen in den einzelnen Antwortkategorien nicht immer möglich. 
Bei einer differenzierten Betrachtung des Wechselwunsches lassen sich für die Haushaltsgröße und das Vorhandensein von Kindern, das Einkommen sowie die Anzahl der Autos im Haushalt statistisch hoch signifikante Unterschiede aufzeigen. Am deutlichsten diskriminierend wirkt jedoch die Einstellung zum Auto, ermittelt über Fragen zur Assoziation von Fahrspaß und Autofahren sowie anhand der Frage, ob die Probanden den Alltag ohne Auto gestalten könnten. Auch die Art des Wohnortes bewirkt deutliche Unterschiede im Antwortverhalten: so antworten beispielsweise Bewohner ländlicher Kreise weniger ablehnend als Großstadtbewohner. Geschlecht, Bildungsstand oder Erwerbsstatus hingegen wirken sich nicht statistisch signifikant auf das Antwortverhalten aus; dies gilt auch für den wahrgenommenen Parkdruck am Wohn- oder Hauptbezugsort. Differenziert nach den Nutzungsszenarien sind eine höhere Skepsis bei Frauen gegenüber dem Vehicle-onDemand hervorzuheben sowie starke Unterschiede im Antwortverhalten gegenüber dem Valet-Parken in Abhängigkeit vom Vorhandensein von Kindern, von der Haushaltsgröße und vom Einkommen.

Die Teilnehmer wurden darüber hinaus für je ein Szenario gefragt, wie sich ihrer Meinung nach die Nutzung eines autonomen Fahrzeugs auf ihre bisherige Verkehrsmittelnutzung auswirken würde. Die Antworten erfolgten dabei für jedes bisherige Verkehrsmittel separat und reichten von ,viel seltener“ (-2) bis hin zu ,viel häufiger“ (+2). Auffallend ist auch hier der große Prozentsatz derjenigen, die nicht von einer Änderung ihres Verhaltens ausgehen: Mit Ausnahme des Taxis und des konventionellen Pkw geben stets zwischen 50 und 64 Prozent der Befragten an, keine Änderung in ihrer Verkehrsmittelnutzung vorauszusehen. Die jeweiligen Mittelwerte der gegebenen Antworten für die einzelnen antizipierten Verlagerungswirkungen sind in Tab. 12.1 darstellt. Ein Mittelwert von 0 ist dabei als Indifferenz zu werten. Die Taxinutzung wird in allen Szenarien als rückläufig eingeschätzt. Bemerkenswert ist, dass das Valet-Parken die stärksten Wechselassoziationen bewirkt - insbesondere bei der Taxinutzung liegt der Mittelwert der Antworten mit -0,78 sehr niedrig.

Tab. 12.1 Auswirkung der Szenarien auf die bisherige Verkehrsmittelnutzung

\begin{tabular}{|l|l|l|l|l|l|}
\hline Szenario & $\begin{array}{l}\text { Ich würde ... } \\
\text { mit öffent- } \\
\text { lichen Ver- } \\
\text { kehrsmitteln } \\
\text { fahren. }\end{array}$ & $\begin{array}{l}\text { Ich würde ... } \\
\text { mit dem Rad } \\
\text { oder zu Fuß } \\
\text { unterwegs } \\
\text { sein. }\end{array}$ & $\begin{array}{l}\text { Ich würde ... } \\
\text { mit dem Zug } \\
\text { unterwegs } \\
\text { sein. }\end{array}$ & $\begin{array}{l}\text { Ich würde ... } \\
\text { mit dem Taxi } \\
\text { fahren. }\end{array}$ & $\begin{array}{l}\text { Ich würde ... } \\
\text { mit einem } \\
\text { konventio- } \\
\text { nellen Pkw } \\
\text { fahren. }\end{array}$ \\
\hline Autobahnpilot & $-0,36$ & $-0,28$ & $-0,35$ & $-0,70$ & 0,04 \\
\hline Valet-Parken & $-0,44$ & $-0,28$ & $-0,51$ & $-0,78$ & $-0,32$ \\
\hline Vollautomat & $-0,33$ & $-0,11$ & $-0,41$ & $-0,74$ & $-0,23$ \\
\hline Vehicle-on-D. & $-0,33$ & $-0,15$ & $-0,35$ & $-0,78$ & $-0,13$ \\
\hline
\end{tabular}

Mittelwerte der Antwortoptionen -2 viel seltener; -1 seltener; 0 gleich häufig; 1 häufiger; 2 viel häufiger 


\subsubsection{Worin sehen die Befragten den spezifischen Vorteil der autonomen Fahrzeuge?}

Eine weitere Frage untersucht, bei welchen Einsatzzwecken oder Wegeeigenschaften sich die Probanden einen Einsatz autonomer Fahrzeuge als besonders hilfreich vorstellen. Die Mittelwerte der Antworten sind in Tab. 12.2 dargestellt. Dass die Unterschiede zwischen den Szenarien mit Ausnahme der Begleitwege stets hoch signifikant sind, kann als Zeichen gewertet werden, dass die Probanden klare Unterscheidungen zwischen den Szenarien vornehmen können. Auffallend sind die vergleichsweise hohen Zustimmungswerte des Vollautomaten auf langen (Überland-)Wegen und Reisen, in geringerem Maße auch bei höheren Besetzungsgraden. Das Valet-Parken wird insbesondere im städtischen Kontext sowie bei Transportaufgaben als hilfreich eingestuft.

Bei einer differenzierten Betrachtung fällt auf, dass männliche Antwortpersonen statistisch signifikant für alle Wegezwecke höhere Zustimmungsraten für das Vehicle-on-Demand angeben; bei Arbeits- und weiten Wegen gilt dies auch für den Autobahnpiloten. Auch lässt sich zeigen, dass der Bildungsstand, gemessen am Vorhandensein des Abiturs, signifikante Auswirkungen auf die Einstellung zum Vehicle-on-Demand auf weiten (Überland-) Wegen, Reisen und bei Wegen in Begleitung aufweist. Die Anwesenheit von Kindern im Haushalt wirkt sich signifikant positiv auf die empfundene Nützlichkeit des Vollautomaten bei Fahrten in die Stadt sowie über Land aus. Bei Bringwegen konnte hingegen kein Zu-

Tab. 12.2 Konstatierte Antworten der Befragten, bei welchen Wegen sie ein solches Fahrzeug besonders hilfreich fänden

\begin{tabular}{|c|c|c|c|c|}
\hline & $\begin{array}{l}\text { Autobahn- } \\
\text { pilot }\end{array}$ & $\begin{array}{l}\text { Valet- } \\
\text { Parken }\end{array}$ & $\begin{array}{l}\text { Vollauto- } \\
\text { mat }\end{array}$ & $\begin{array}{l}\text { Vehicle-on- } \\
\text { Demand }\end{array}$ \\
\hline $\begin{array}{l}\text {...wenn ich zur Arbeit oder zur Aus- } \\
\text { bildung fahre. }\end{array}$ & $-1,0$ & $-0,4$ & $-0,2$ & $-0,6$ \\
\hline $\begin{array}{l}\text {...wenn ich einkaufen fahre oder } \\
\text { Erledigungen nachgehe. }\end{array}$ & $-1,2$ & 0,2 & $-0,3$ & $-0,4$ \\
\hline ...wenn ich Personen bringe oder abhole. & $-0,8$ & $-0,4$ & $-0,1$ & $-0,5$ \\
\hline ...wenn ich zur Freizeitgestaltung fahre. & $-0,8$ & $-0,4$ & $-0,4$ & $-0,7$ \\
\hline $\begin{array}{l}\text {...wenn ich Reisen oder Ausflüge unter- } \\
\text { nehme. }\end{array}$ & 0,1 & $-0,1$ & 0,4 & $-0,2$ \\
\hline ...wenn ich lange unterwegs bin. & 0,4 & $-0,2$ & 0,8 & 0,2 \\
\hline ...wenn ich in Begleitung bin. & $-0,4$ & $-0,4$ & 0,0 & $-0,4$ \\
\hline ...wenn ich in der Stadt unterwegs bin. & $-1,1$ & 0,5 & $-0,1$ & $-0,4$ \\
\hline ...wenn ich über Land fahre. & $-0,4$ & $-1,0$ & 0,3 & $-0,3$ \\
\hline ...wenn ich Gepäck dabei habe. & $-0,9$ & 0,3 & $-0,4$ & $-0,5$ \\
\hline
\end{tabular}

Mittelwerte der Antwortoptionen -2 viel seltener; -1 seltener; 0 gleich häufig; 1 häufiger; 2 viel häufiger 
sammenhang festgestellt werden. Bei weiten Wegen und Fahrten in die Stadt zeigen sich für das Valet-Parken Antwortunterschiede in Abhängigkeit von der Haushaltsgröße sowie der Anwesenheit von Kindern. Der Erwerbsstatus ${ }^{2}$ bedingt fast ausschließlich beim ValetParken Unterschiede in den Antworten auf Arbeitswegen, weiten Wegen sowie Freizeitwegen; bei letzteren gilt dies zusätzlich auch für die Einkommenshöhe.

\subsubsection{Was machen wir heute und zukünftig unterwegs?}

Die Möglichkeit, während der Fahrt einer anderen Betätigung nachgehen zu können, zählt zu den hauptsächlichen Eigenschaften des automatisieren Fahrens aus Nutzungssicht. Gleichzeitig stellen Zeitkosten einen Haupttreiber der Verkehrsmittelwahl in Modellen dar. Abschließend soll daher ein Blick auf Ergebnisse der Befragung hinsichtlich der bisherigen und vielleicht auch zukünftigen Zeitnutzung geworfen werden.

Die Probanden wurden zunächst gefragt, welchen Beschäftigungen sie in der Regel bei Fahrten mit dem öffentlichen Nahverkehr, der Bahn oder dem Auto nachgehen. Die mit Abstand meistgenannten Betätigungen im öffentlichen Verkehr sind der Genuss der Landschaft und der Fahrt: 50 Prozent der Befragten geben an, dies im ÖV häufig oder immer zu tun, in der Bahn 66 Prozent. Ähnlich beliebt ist die Unterhaltung mit Begleitpersonen oder anderen Passagieren (ÖV: 42 Prozent, Bahn: 49 Prozent). Es folgen das Hören von Musik, Lesen oder die Entspannung als oft genannte Tätigkeiten. Rund 77 bzw. 69 Prozent der Befragten geben an, nie im Nah- oder Fernverkehr zu arbeiten; knapp 6 bzw. 8 Prozent arbeiten häufig oder immer. Die Antworten zur Arbeit unterwegs unterscheiden sich insbesondere im Zug statistisch signifikant nach Geschlecht, Einkommen, Bildungsstand, Haushaltsgröße sowie dem Vorhandensein von Kindern im Haushalt. So geben 74 Prozent der Frauen und 63 Prozent der Männer an, im Zug nie zu arbeiten; die Wahrscheinlichkeit, im Zug häufig oder immer zu arbeiten, verdoppelt sich bei einem Nettohaushaltseinkommen von über 2600 Euro gegenüber der Vergleichsgruppe auf rund 10 Prozent.

Momentane Hauptbetätigung im Pkw ist naturgemäß die Konzentration auf die Fahrt und die Route. Rund 80 Prozent hören dabei häufig oder immer Musik; rund zwei Drittel unterhalten sich, über die Hälfte der Befragten genießt unterwegs häufig oder immer die Fahrt und die Landschaft. 7 Prozent der Befragten geben an, manchmal im Auto zu arbeiten.

Betrachtet man die von den Befragten wahrgenommenen besonderen Vorteile autonomen Fahrens im Hinblick auf die Möglichkeit einer alternativen Zeitnutzung während der Fahrt, so werden vor allem die Möglichkeit zur Unterhaltung sowie des Landschaftsgenusses genannt - den bereits jetzt liebsten Beschäftigungen im Auto. Exemplarisch sind in Abb. 12.2 die Antworten zum vollautomatisierten Fahrzeug dargestellt. Auffallend ist der geringe Anteil derjenigen, die einen Vorteil in der Möglichkeit sehen, unterwegs arbeiten zu können; beim Autobahnpiloten ist es weniger als ein Viertel der Befragten.

2 Beim Erwerbstatus wurde zwischen Vollzeit-, Teilzeit- sowie sonstiger Beschäftigung unterschieden. 


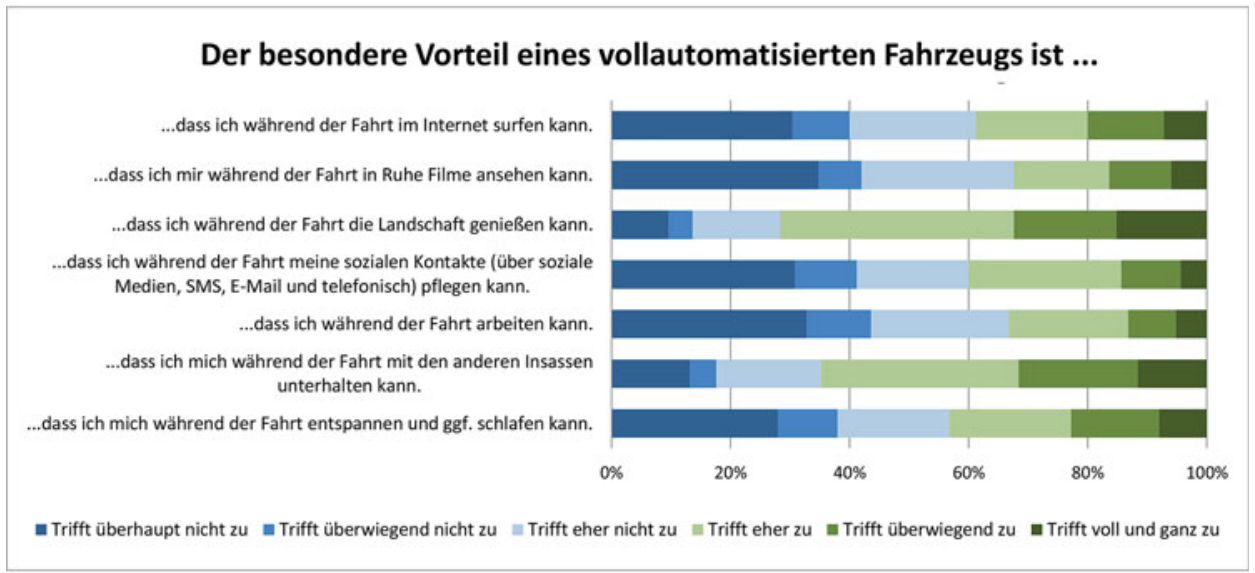

Abb. 12.2 Konstatierte Vorteile des vollautomatisierten Fahrzeugs

\subsection{Autonome Fahrzeuge in der Nachfragemodellierung: Möglichkeiten und Grenzen einer Integration}

Die Analyse der möglichen Wirkungen der einzelnen Nutzungsszenarien zeigt deutlich die Notwendigkeit einer Differenzierung bei der Integration autonomer Fahrzeuge in die Nachfragemodellierung auf. Während es sich sowohl beim Autobahnpiloten als auch bei der Funktion des Valet-Parkens in weiten Teilen um spezielle, temporär beschränkte Sonderformen eines ansonsten weitgehend gleich bleibenden Autofahrens bzw. Fahrzeugs handelt, ist mit der Einführung vollautomatisierter Fahrzeuge ein quasi neues Verkehrsmittel oder mit der Einführung einer Vehicle-on-Demand-Flotte möglicherweise ein komplett neuartiges Mobilitätsangebot verbunden.

Die sicherlich größte Herausforderung bei der Abbildung autonomen Fahrens in der Nachfragemodellierung besteht darin, eine stärkere Differenzierung des Individualverkehrsmittels „Auto“ zu ermöglichen. Der klassischen Beschreibung dieser Verkehrsmitteloption anhand der Hauptkriterien Fahrzeit- und Nutzungskosten sowie einer oftmals nicht weiter spezifizierten Präferenz der Entscheidenden mangelt es vor allem an der Möglichkeit, die Rolle des Fahrers und des Besitzstandes angemessen zu berücksichtigen. Unterscheidungen zwischen Fahren und Gefahrenwerden, dem Fahren des eigenen oder eines beliebigen Fahrzeuges sind Voraussetzung einer adäquaten Abbildung. Nur so ist es möglich, sowohl die Rolle des Fahrers von der des Beifahrers oder des vom Vollautomaten Chauffierten zu unterscheiden. Ebenso notwendig ist eine Differenzierung zwischen dem klassischen Pkw, einem vollautonomen Fahrzeug, einem Taxi oder einem geliehenen Vehicle-on-Demand. Der Wunsch einer Integration autonomer Fahrzeuge in die Modellierung geht somit direkt einher mit der Notwendigkeit, die einzelnen zur Verfügung stehenden Alternativen differenzierter beschreiben zu können. Gerade die fließenden, vom Nutzer 
und seiner Fahrt abhängigen Übergänge vom Fahrer zum Quasi-Beifahrer beim Autobahnpiloten und beim Vollautomaten stellen hier eine besondere Herausforderung dar.

Die Reisezeit ist einer der einflussreichsten Treiber der Verkehrsmittelwahl in bestehenden Verkehrsmodellen. Bei der Modellierung des öffentlichen Verkehrs wird häufig zwischen der Onboard- oder eigentlichen Reisezeit, der Wartezeit sowie den Zugangs- und Abgangszeiten unterschieden, sodass auch unterschiedliche Bewertungen der Zeitkomponenten möglich sind. Bisher erfolgt jedoch weder hier noch bei anderen Verkehrsmitteln eine vertiefte Betrachtung der im Fahrzeug verbrachten Zeit. Die mit der Einführung autonomer Fahrzeuge verbundenen Erwartungen, die Zeit im Fahrzeug subjektiv sinnhafter verbringen zu können, zeigt daher deutlich die Notwendigkeit einer Unterscheidung in den Modellen auf. Die Distanz bzw. Dauer der Fahrt und damit auch der potenziell nutzbare Fahrtenanteil rücken hier ebenso in den Fokus wie die Frage, welche alternativen Betätigungen angestrebt werden oder mit wem die Fahrt durchgeführt wird.

Doch nicht nur die Zeit im Fahrzeug ist Änderungen unterworfen, besteht doch die auffälligste vom Valet-Parken bedingte Änderung in einer Verringerung des Nutzungsaufwandes am Anfang und am Ende der Fahrt. Gleiches gilt für das Vehicle-on-Demand. Auch hier zeigt sich die Notwendigkeit, die in den Modellen oftmals sehr pauschale Betrachtung der mit der Wahl eines Verkehrsmittels einhergehenden Reisezeit weiter zu differenzieren. Als konkrete Beispiele seien hier die Wartezeiten auf ein Taxi oder die Zugangszeiten zum eigenen oder geliehenen Pkw genannt.

Ein Aspekt, der sich in vereinfachter Form durch eine Modifikation der Reisezeiten vergleichsweise leicht in die Modelle integrieren lässt, besteht in der Verkürzung der Reisezeiten, die sich direkt aus dem Fehlen von Staus oder störenden Parksuchverkehren ergeben könnte. Problematischer ist die zumeist fehlende Abbildung der Wirkung einer Verlässlichkeitssteigerung der zeitlichen Prognosen von Reisezeiten auf die Bewertung des Pkw als Verkehrsmittelalternative. Die Komfortsteigerung, die sich aus neuen Funktionen wie dem Valet-Parken oder dem Autobahnpiloten ergeben, der eventuelle Sicherheitsanstieg oder auch die erhöhte Flexibilität der Fahrzeugausstattung sind weitere Beispiele relevanter subjektiver Kriterien, die bei der bisherigen Betrachtung der Verkehrsmittelwahl kaum Berücksichtigung finden. Die Problematik, subjektive Aspekte zu erfassen, in ihrer Wirkung zu quantifizieren und bei der Verkehrsmittelwahl als Faktoren zu berücksichtigen, gilt jedoch nicht nur für die Eigenschaften der Verkehrsmittel, sondern mindestens ebenso für die Beschreibung der Nutzer. Insbesondere der empfundene Fahrspaß, das Empfinden des eigenen Fahrvermögens, die Bereitschaft zur Abgabe der Fahrfunktion, die Aversion gegenüber einem etwaigen Kontrollverlust oder das Vertrauen in die Technik sind Beispiele von Faktoren, die gerade bei autonomen Fahrzeugen verstärkte Relevanz bei der Beurteilung erlangen (s. Kap. 29) und somit vermehrt in den Fokus der Modellierung rücken sollten.

Die Identifikation von Nutzergruppen, die sich hinsichtlich ihrer Bewertung verschiedener Verkehrsmitteloptionen unterscheiden, ist eines der wichtigsten Fundamente zur Beschreibung der Personen in den Modellen. Sowohl die literaturbasierten Wirkungsanalysen als auch die Ergebnisse der Befragung weisen deutlich darauf hin, dass sich die realen oder wahrgenommenen Vorteile autonomer Fahrzeuge nicht nur je nach Szenario, 
sondern auch je nach Einstellungen und soziodemografischen Eigenschaften unterscheiden. Neben den klassischen Attributen Geschlecht, Erwerbsstatus, Alter und Führerschein zeigten sich je nach Nutzungsszenario vor allem die Haushaltsgröße, das Vorhandensein von Kindern im Haushalt, das Bildungsniveau sowie das Haushaltseinkommen als relevante Unterscheidungsfaktoren. Im Zuge einer Abbildung autonomer Fahrzeuge wäre hier sicherlich eine Erweiterung der in den Modellen verwendeten Attribute zur Beschreibung der Personen, vor allem aber auch des Haushaltskontextes zu diskutieren. Darüber hinaus gilt auch hier, dass die Berücksichtigung von Einstellungsfaktoren wie die Haltung gegenüber verschiedenen Verkehrsmitteln, Routinen oder auch zeitliche Eingebundenheit kaum erfolgt.

Für die Verkehrsmittelwahl besonders relevante Personen- und Haushaltsattribute sind der Besitz eines Führerscheins sowie der Zugang zu einem Pkw, in der Regel ausgedrückt durch die Anzahl der Pkw im Haushalt oder auch die tatsächliche Verfügbarkeit am Stichtag der Verhaltenserhebung. In der makroskopischen Modellierung handelt es sich bei der Pkw-Verfügbarkeit um das gängige Kriterium, anhand dessen die Nutzersegmentierung vorgenommen und die Aufkommensraten bestimmt werden. Bei der Verkehrsmittelwahl selbst wird dann von einer generellen Verfügbarkeit des Pkw ausgegangen. Mikroskopische Verkehrsmodelle erlauben es, neben dem Führerscheinbesitz auch die Beschränkungen der tatsächlichen Verfügbarkeit des Pkw zur Wahlsituation zu berücksichtigen - sei es durch Nutzungskonkurrenz im Haushalt oder vor allem im Verlauf der Wegekette. Systeme wie das Vehicle-on-Demand brechen diese Verfügbarkeitsbeschränkung systematisch auf diese Art von Pkw kann theoretisch jederzeit von jeder Person als neue Verkehrsmitteloption gewählt werden. Ebenso wie bei der Abbildung von Carsharing-Systemen gilt es hier also nicht zuletzt, die räumlichen und sozialen Kriterien der Pkw-Verfügbarkeit bei der Modellierung zu überdenken.

Die Berücksichtigung von Raum- und Kontexteigenschaften sowie die Differenzierung der möglichen Einsatzzwecke oder Wegezwecke waren in Abschn. 12.3.2 als weitere Kriterien der Verkehrsmittelwahl in den Modellen aufgeführt worden. Insbesondere beim Nutzungsszenario des Valet-Parkens erschließt sich intuitiv die hohe Bedeutung des Raumes für die wahrgenommene Nützlichkeit der Option. Zwar ist der Detaillierungsgrad in den Nachfragemodellen in der Regel hoch genug, um Aussagen über die Siedlungsstruktur, die generelle Flächennutzungsstruktur oder auch die Bebauungsdichte treffen zu können. Die Bebauungsstruktur - nicht zuletzt ausschlaggebend dafür, ob genügend oder gar private Stellplätze vorhanden sind - ist in der Regel nicht abgebildet. Detaillierte Abbildungen der Verfügbarkeit von Parkplätzen, des Parkdruckes oder auch der damit assoziierten Kosten sowie der Zugangs- und Abgangszeiten stellen eine Seltenheit dar (s. Abschn. 12.3.2). Für eine adäquate Abbildung des Valet-Parkens lässt sich hier generell Handlungsbedarf feststellen.

Das Vehicle-on-Demand ist ebenfalls ein Beispiel, in dem die Raumstruktur maßgeblich über die Nutzung und die Konkurrenzsituation zu bestehenden Verkehrsmittelangeboten bestimmt wird. So gilt es im städtischen Raum vornehmlich als Alternative zum privaten Pkw, dem Taxi oder dem öffentlichen Verkehr, im ländlichen Raum wird es vor allem als 
möglicher Zubringerdienst und somit als Ergänzung zum Angebot des öffentlichen Verkehrs gesehen. Die Aufgabe der Modellierung ist es somit, sinnvolle Umsetzungskonzepte und Verfügbarkeiten zu antizipieren und in die Abbildung des intermodalen Verkehrsangebotes zu integrieren. Weitere erstrebenswerte Verbesserungen bei der Abbildung des Verkehrsangebotes betreffen die Abbildung der Parksuchverkehre sowie die Identifikation der Streckenabschnitte, die vorrangig für den Einsatz entsprechender Automatisierungen geeignet sind.

Insbesondere das Nutzungsszenario des Vehicle-on-Demand macht deutlich, welche grundsätzlichen Auswirkungen autonome Fahrzeuge auf unseren Fahrzeugbesitz und damit unsere alltäglichen Mobilitätsentscheidungen haben könnten. Der Abbildung und Quantifizierung kausaler Zusammenhänge zwischen dem Besitz oder der Zugangsoption zu einem Fahrzeug und seinen wichtigsten Eigenschaften - beispielsweise Ausstattung mit ValetParken oder einer vollautomatischen Fahrfunktion - einerseits und den angebotsseitigen, räumlichen und soziodemografischen Eigenschaften des jeweiligen Haushaltes andererseits kommt damit eine verstärkte Bedeutung zu. Zwar wird die klassische Herangehensweise, lediglich den Besitz eines Durchschnittsautos als Eingangsgröße in die Modelle zu berücksichtigen, insbesondere unter dem Gesichtspunkt der Emissionsberechnung mehr und mehr durch vorgelagerte Modelle abgelöst, indem Antriebsart, Größenklasse, Preissegment, Anzahl der Sitzplätze oder Transportmöglichkeiten der in den Haushalten verfügbaren Pkw einbezogen werden. Unterstützungsfunktionen wie das Valet-Parken spielen dabei aber ebenso wenig eine Rolle wie die Alternative einer Carsharing-Mitgliedschaft oder die Abschaffung eines Pkw aufgrund einer guten Versorgung mit Verkehrsmittelalternativen. Doch nicht nur die Frage, wer den Besitz eines eigenen Autos durch das Leihen oder das Gefahrenwerden substituieren könnte, bleibt so mit den herkömmlichen Mitteln der Modellierung unbeantwortet. Ist davon auszugehen, dass insbesondere die Kombination aus eigenem Pkw und verschiedenen Vehicles-on-Demand eine flexible Anpassung des eingesetzten Fahrzeuges auf die jeweilige Nutzungssituation fördert, so zeigt sich auch hier dringlicher Bedarf bei der Erhebung entsprechender Daten, ihrer Analyse und der nachfolgenden Erweiterung der Modellierung.

\subsection{Zusammenfassung und Ausblick}

Es wird allgemein erwartet, dass autonome Fahrzeuge unterschiedlicher Ausprägung in naher Zukunft Teil unserer Alltagsmobilität sein werden. Die Integration entsprechender Verkehrsangebote in die Verkehrsmodellierung, eines der wichtigsten Instrumente der Planungspraxis, stellt daher gleichermaßen Notwendigkeit und Herausforderung dar: Bestehende empirische Arbeiten zu den erwarteten Auswirkungen zeigen sich bisher vorrangig technikorientiert; gleichzeitig ist der Blick in die Zukunft naturgemäß unsicherheitsbehaftet und die Wirkungsweise neuer Technologien nur schwer abzuschätzen.

Betrachtet man die einzelnen Nutzungsszenarien hinsichtlich ihrer möglichen Wirkungen auf die Verkehrsmittelwahl, so zeigt sich deutlich die Notwendigkeit der Differenzierung. 
Sowohl das Valet-Parken als auch der Autobahnpilot werden vorrangig mit einem geringfügig modifizierten „,normalen“ Pkw verbunden, der in speziellen Situationen einen Vorteil bietet: beim Valet-Parken der verbesserte Zugang zum Pkw bzw. der Wegfall der Parkplatzsuche, beim Autobahnpiloten die optionale Abgabe der Fahrfunktion auf ausgewählten Strecken oder in speziellen Fahrsituationen. Mit der Einführung vollautomatisierter Fahrzeuge verbindet sich die Vorstellung einer substanziellen Verbesserung der Bewertung der Reisezeit im Fahrzeug und einer gleichzeitigen Senkung der Hindernisse für die Nutzung des Pkw. Tiefgreifende Auswirkungen auf das Gesamtverkehrssystem lassen sich vor allem für das Szenario einer weitreichenden Verfügbarkeit von Vehicles-on-Demand aufzeigen: Individualmobilität wird unabhängig von der Fahrertüchtigung oder dem Besitz eines Fahrzeuges möglich. Voraussichtlich wird dieses fahrerlose Carsharing in Städten neben einer deutlichen Senkung des Pkw-Besitzes zu einer Reduktion der Bringwege und der Taxinutzung führen; auf dem Land könnte es neue Zugänge zum öffentlichen Verkehr erschließen.

Mithilfe einer Onlinebefragung wurde untersucht, ob bzw. im Austausch gegen welche bisher genutzten Verkehrsmittel sich die Befragungsteilnehmer vorstellen könnten, ein autonomes Fahrzeug in der Form verschiedener Nutzungsszenarien zu nutzen. Neben einer generellen, mit der Konkretisierung in den einzelnen Szenarien sogar steigenden Nutzungsskepsis ist der große Einfluss der momentanen Einstellung zum Auto auffallend. Besonders starke Nutzungsrückgänge werden für das Taxi antizipiert. Spezifische Vorteile autonomer Fahrzeuge werden vor allem bei langen Fahrten und im Falle des Valet-Parkens im städtischen Kontext gesehen. Der Genuss der Fahrt und der Landschaft sowie die Unterhaltung mit Mitfahrenden sind momentan die bevorzugten Tätigkeiten in Nah- und Fernverkehr; mehr als zwei Drittel geben an, nie unterwegs zu arbeiten. Während der Autofahrt gehören das Musikhören und die Unterhaltung zu den am weitesten verbreiteten Aktivitäten. Bei der Frage nach der zukünftigen Zeitnutzung beim automatisierten Fahren stehen auch hier Landschaftsgenuss und Unterhaltung im Vordergrund der Nennungen.

Nicht alle Faktoren, die sich als maßgeblich für die Bewertung autonomer Fahrzeuge erweisen, lassen sich in Verkehrsmodellen mit ihrer vereinfachten Darstellung der Kausalzusammenhänge bei der Verkehrsentstehung abbilden. Insbesondere nicht-rationale, ,weiche" Wahrnehmungs- und Bewertungsfaktoren wie der Fahrspaß oder aber der Wunsch bzw. die Ablehnung, die Fahraufgabe abzugeben, lassen sich empirisch nur schwer und aufwendig fassen und in Modellen berücksichtigen. Sowohl die empirische Basis als auch die modellseitige Umsetzung zeigen hier Erweiterungsbedarf und -potenziale.

Die hauptsächliche Herausforderung bei der Integration autonomer Fahrzeuge liegt jedoch in der bisher nur rudimentären Unterscheidung des „Autos“ in den Modellen. Auch hier gilt es, empirische Grundlagen ebenso wie die Abbildungsmöglichkeiten in den Modellen zu verbessern und gleichzeitig die Rolle der Fahraufgabe und des Fahrzeugbesitzes bei der Verkehrsmittelwahl zu unterstreichen. Ziel ist, eine deutlichere Unterscheidung zwischen Fahren und Gefahrenwerden, einem vollständig automatisierten und einem nur bei Wunsch unterstützenden Fahrzeug, dem eigenen und einem geliehenen Pkw oder auch einem Taxi zu ermöglichen. Hier offenbart sich zeitgleich die Notwendigkeit, Fahrzeugbesitz als feste Inputgröße in der Modellierung zu überdenken. 
Die Möglichkeit einer geänderten Zeitnutzung ist neben der Sicherheit einer der meistgenannten Vorteile einer Automatisierung des Fahrens. Gleichzeitig wirken Zeitkosten als Haupttreiber bei der Verkehrsmittelwahl in Verkehrsnachfragemodellen. Modellseitig ist bisher allerdings in der Regel keine Unterscheidung der Reisezeit vorgesehen. Unterscheidungen zwischen ,sinnvoll“ und „,nutzlos“ verbrachter Zeit, zwischen aktivem Fahrspaß oder lästigem Staustehen, zwischen produktiver Arbeit oder entspannendem Musikhören sind derzeit nicht möglich. Eine derartige Erweiterung der Modelle ist im Prinzip nicht schwierig - zunächst unabhängig davon, welche Alternativbetätigung tatsächlich angestrebt wird. Doch wird erneut deutlich, dass die empirische Basis, auf der die Modellierung aufbaut, zu große Lücken aufweist, um eine adäquate Abbildung automatisierten Fahrens gewährleisten zu können.

\section{Literatur}

1. Autoscout 24: Unser Auto von morgen: Studie zu den Wünschen der Europäer an das Auto von morgen. (2012)

2. Bamberg, S., Hunecke, M., Blöbaum, A.: Social context, personal norms and the use of public transportation: Two field studies. J. Environ. Psychol. 27, 190-203 (2007)

3. Becker, J.: Fahrplan in die Zukunft, Süddeutsche Zeitung, 18.1.2014, S.40. (2014)

4. Bhat, C.R., Sardesai, R.: The impact of stop-making and travel time reliability on commute mode choice. Transp. Res. Part B Methodol. 40, 709-730 (2006)

5. Bates, J.J.: History of Demand Modelling. In: Hensher, D.A. and Button, K.J. (eds.) Handbook of Transport Modelling. pp. 11-34. Elsevier (2012)

6. Buehler, R.: Determinants of transport mode choice: a comparison of Germany and the USA. J. Transp. Geogr. 19, 644-657 (2011)

7. Castiglione, J., Bradley, M., Gliebe, J.: Activity-Based Travel Demand Models : A Primer. (2014)

8. Continental: Continental Mobilitätsstudie 2013. (2013)

9. Cervero, R., Kockelman, K.: Travel demand and the 3Ds: Density, diversity, and design. Transp. Res. Part D Transp. Environ. 2, 199-219 (1997)

10. Davidson, W., Donnelly, R., Vovsha, P., Freedman, J., Ruegg, S., Hicks, J., Castiglione, J., Picado, R.: Synthesis of first practices and operational research approaches in activity-based travel demand modeling. Transp. Res. Part A Policy Pract. 41, 464-488 (2007)

11. Fagnant, D.J., Kockelman, K.M.: Preparing a Nation for Autonomous Vehicles: Opportunities, Barriers and Policy Recommendations. Eno Cent. Transp. (2013)

12. Flade, A., Wullkopf, U.: Theorien und Modelle zur Verkehrsmittelwahl. Darmstadt (2002).

13. Giesel, F., Lenz, B.: Wirkung von E-Car-Sharing-Systemen auf Mobilität und Umwelt in urbanen Räumen, unveröffentlichte WiMobil-Projektpräsentation. (2014)

14. Giesel, F., Nobis, C., Lenz, B.: Carsharing as a Driver of Sustainable Urban Mobility? An Analysis of User Structure and Motivation, unveröffentlichte Präsentation auf der AAG Conference in Tampa, USA. (2014)

15. Glotz-Richter, M., Loose, W., Nobis, C.: Car-Sharing als Beitrag zur Lösung von städtischen Verkehrsproblemen. Int. Verkehrswes. 59, 333-337 (2007).

16. Kahneman, D., Krueger, A.B.: Development in the Measurement of Subjective Well-Being. J. Econ. Perspect. 20, 3-24 (2006)

17. KPMG: Self-Driving Cars: Are We Ready? (2013) 
18. Kutter, E.: Modellierung für die Verkehrsplanung. Theoretische, empirische und planungspraktische Rahmenbedingungen. ECTL Working Paper 21, TU Hamburg-Harburg. (2003)

19. Litmann, T.: Ready or waiting? Traffic Technol. Int. 37-42 (2014)

20. Maier, G., Weiss, P.: Modelle diskreter Entscheidungen: Theorie und Anwendung in den Sozialund Wirtschaftswissenschaften. Springer (1990)

21. Nally, M.G., Rindt, C.: The activity-based approach. In: Hensher, D.A. and Button, K. (eds.) Handbook of Transport Modelling. pp. 53-69. Elsevier (2012)

22. McNally, M.G.: The Four-Step Model. In: Hensher, D.A. and Button, K. (eds.) Handbook of Transport Modelling. pp. 35-54. Elsevier (2012)

23. Munsch, E.: Autonomes Fahren: Platz sparen mit dem Bordcomputer, http://www.zeit.de/mobilitaet/2014-05/autonomes-fahren-feldversuch-schweden. (2014). Letzter Zugriff 28.6. 2014

24. Ortùzar, J., Willumsen, L.G.: Modelling Transport, 3. Auflage. Wiley, Chichester (2005)

25. Ory, D.T., Mokhtarian, P.L.: When is getting there half the fun? Modeling the liking for travel. Transp. Res. Part A Policy Pract. 39, 97-123 (2005)

26. Rinspeed: Rinspeed - Creative think tank for the automotive industry. Where the future is reality - today. http://www.rinspeed.eu/aktuelles.php?aid=15. (2014). Letzter Zugriff 28.6. 2014

27. Scheiner, J., Holz-Rau, C.: Travel mode choice: affected by objective or subjective determinants? Transportation (Amst). 34, 487-511 (2007)

28. Scheiner, J., Holz-Rau, C.: Gendered travel mode choice: a focus on car deficient households. J. Transp. Geogr. 24, 250-261 (2012)

29. Schwanen, T., Banister, D., Anable, J.: Rethinking habits and their role in behaviour change: the case of low-carbon mobility. J. Transp. Geogr. 24, 522-532 (2012)

30. Silberg, G., Wallace, R., Matuszak, G.: Self-driving cars: The next revolution. KPMG and Center for Automotive Research. (2012)

31. Simma, A., Axhausen, K.W.: Commitments and Modal Usage: Analysis of German and Dutch Panels. Transp. Res. Rec. J. Transp. Res. Board. 1854, 22-31 (2003)

32. Sokolow, A.: Autonome Autos: Autobranche vs. Google, http://m.heise.de/newsticker/meldung/ Autonome-Autos-Autobranche-vs-Google-2072050.html. (2013). Letzter Zugriff 28.6.2014

33. Steg, L.: Car use: lust and must. Instrumental, symbolic and affective motives for car use. Transp. Res. Part A Policy Pract. 39, 147-162 (2005)

34. Train, K.E.: A comparison of the predictive ability of mode choice models with various levels of complexity. Transp. Res. Part A Gen. 13, 11-16 (1979)

35. Walker, J.L.: Beyond Rationality in Travel Demand Models. ACCESS Mag. 1, https://escholarship.org/uc/item/1x04f3k3. (2011). Letzter Zugriff 28.6.2014

36. Wardman, M.: Public transport values of time. Transp. Policy. 11, 363-377 (2004)

37. Willumsen, L.G.: Forecasting the impact of Self-Driving-Cars. What to do about them in our models and forecasts. Vortrag auf der 2013 Citilab Asia User Conference, Karon, Thailand, unveröffentlicht. (2013) 